\title{
Enhanced $\beta$-carotene biosynthesis in recombinant Escherichia coli harboring the bottom portion of mevalonate pathway of Enterococcus faecium VTCC-B-935 isolated in Vietnam
}

\begin{abstract}
$\beta$-carotene is a carotenoid pigment which has commercial value. Biosynthesis of this pigment using recombinant $E$. coli has been reported. However, improvement of yield is remained necessary. In this case report, bottom portion of mevalonate partway was employed for enhancing metabolic flow to isopentenyl diphosphate, the building block of all carotenoid. Three genes including mvaK1, mvaK2, and mvaD were cloned from Enterococcus faecium VTCC-B-935 isolated in Vietnam and placed into pET28a(+) vector resulted in pET28-K1K2D. Co-expression of this vector with pRSET-1EIBY which contains five enzymes responsible for biosynthesis of $\beta$-carotene in $E$. coli BL21 (DE3) resulted in approximately three fold higher yield of the compound. The high copy number vector pRSET-A showed better performance in production of $\beta$-carotene over low copy pET-22b(+). Addition of glycerol significantly enhanced E. coli cell growth and $\beta$-carotene biosynthesis.
\end{abstract}

Keywords: $\beta$-carotene, enterococcus faecium VTCC-B-935, mvaK1, mvaK2, mvaD, mevalonate pathway
Volume 3 Issue 4 - 2017

\author{
Vu Hoai Nam, ${ }^{2}$ Huyen Trang $\mathrm{HT},{ }^{2}$ Thu Huong \\ LT,' Duong Van CuongI, \\ 'Department of Biotechnology and Food Technology, Thai \\ Nguyen University of Agriculture and Forestry,Vietnam \\ ${ }^{2}$ Division of Molecular Biology, Thai Nguyen University, Vietnam
}

Correspondence: Duong Van Cuong, Faculty of Biotechnology and Food Technology, Thai Nguyen University of Agriculture and Forestry and Division of Molecular Biology, Institute of Life Sciences, Thai Nguyen University,Vietnam,

Email duongvancuong@tuaf.edu.vn

Received: June 07, 2016 | Published: July 05, 2017
Abbreviations: IPP, isopentenyl diphosphate; MEP, 2-C-methyl-d-erythritol-4-phosphate; MVA, mevalonate; DMAPP, dimethylallyl diphosphate; FPP, farnesyl diphosphate; GGPP, geranylgeranyl diphosphate; G3P, glyceraldehyde-3-phosphate

\section{Introduction}

$\beta$-carotene, one of the carotenoid compounds, was found mainly in plants. Because of it's highly antioxidant activity, this compound was shown to have good effects to human health. It is the precursor of vitamin A which is critically required for human. ${ }^{1}$ Due to good health effects and yellow color, $\beta$-carotene is widely used in industries including nutraceuticals, pharmaceuticals, food colorants, cosmetics, and animal feed additives. However, production of natural $\beta$-carotene is not sufficient to afford demand of the market. Currently, more than $90 \%$ of commercially available $\beta$-carotene is chemically synthesized. ${ }^{2}$ This fact leads a number of research groups to the trend of seeking for alternative sources of natural $\beta$-carotene. Natural carotenogenic microorganisms, e.g. Blakeslea trispora, Rhodotorula glutinis, and Dunaliella salina have been used for fermentation to produce $\beta$-carotene. ${ }^{3-5}$

The availability of carotenoid genes from natural carotenogenic organisms leads to another strategy of natural carotenoid biosynthesis. Carotenoid genes were cloned and introduced into non-carotenogenic organism, Escherichia coli, for production of carotenoid, including $\beta$-carotene. ${ }^{6-19} E$. coli is considered as one of the ideal hosts because of its convenience genetic engineering system and fast growth. However, to date, the production of $\beta$-carotene is still unable to support industrial needs because of insufficient yield and stability. Therefore, attempts are required for improvement of production of carotenoid in general and $\beta$-carotene in particular. Biosynthesis of $\beta$-carotene was affected by a number of factors. In our study, we focus in

i. The different expression vector backbones

ii. Origin of foreign genes

iii. Availability and balance of isopentenyl diphosphate (IPP) which is the building blocks of carotenoids, and

iv. Effect of additional carbon sources

Biosynthesis partway of $\beta$-carotene is indicated in Figure 1. DMAPP and IPP, the building blocks of carotenoid are synthesized via 2-C-methyl-d-erythritol-4-phosphate (MEP) pathway which is autonomous in our chosen host $E$. coli. However, in theory the natural yield of these precursors is only sufficient for natural need of $E$. coli in normal growth conditions which is far less than that required once the organism is used as a microbial factory to produce $\beta$-carotene. To address this issue, addition of the exogenous mevalonate (MVA) pathway was shown as a reasonable strategy. ${ }^{6,12,18,20,21}$ The MVA pathway is divided into two portions, the upper (from acetyl-CoA to MVA) and the lower (from MVA to DMAPP and IPP).

Natural E. coli harbors MEP pathway that enable biosynthesis of FPP from G3P and pyruvate, as well as IPP isomerase catalyze the two-way conversion of IPP and DMAPP, the precursors of carotenoid (yellow part). These precursors could also be synthesized by the mevalonate pathway which is exogenous to E. coli. In this design, the bottom portion of MVA pathway from mevalonate to IPP was recruited from $E$. faecium VTCC-B-935 isolated in Vietnam (green part). IPP is subsequently used as building blocks for the process of $\beta$-carotene synthesis including four steps catalyzed by exogenous $\mathrm{crt}$ 
genes derived from $P$. ananatis. For better balancing between the two isoforms IPP and DMAPP, in addition to the endogenous idi gene positioned in the genome of $E$. coli host, another copy of this gene was introduced into the carotenogenic vector for co-over expression with $c r t$ genes (blue part).

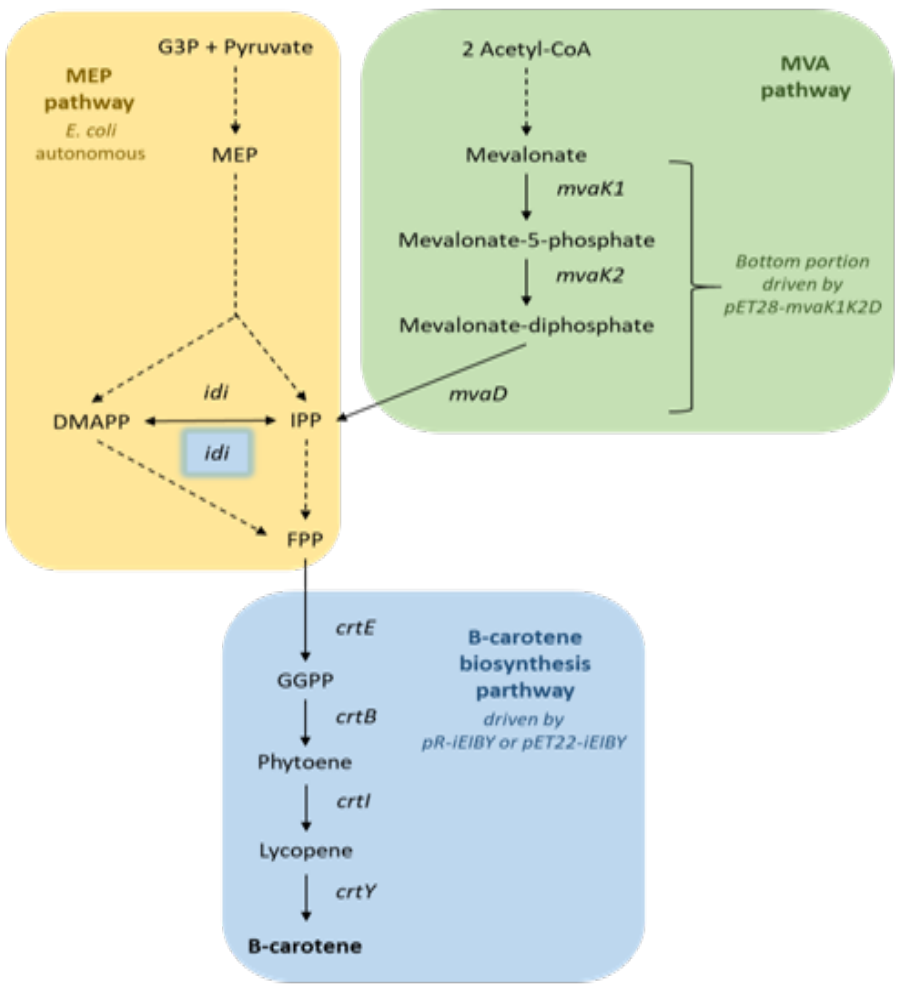

Figure I Biosynthesis pathways of $\beta$-carotene in recombinant $E$. coli engineered in this study.

G3P, glyceraldehyde-3-Phosphate; MEP, 2-C-methyl-d-erythritol-4-phosphate; IPP, isopentenyl diphosphate; DMAPP, dimethylallyl diphosphate; FPP, farnesyl diphosphate; GGPP, geranylgeranyl diphosphate

In this case report, we first examine the difference of expression vector backbones. In our previous study, three genes including $c r t E$, $\operatorname{crt} B$, and $c r t I$ were cloned from Pantoea ananatis for lycopene biosynthesis, and $i d i$ was cloned from $E$. coli for better balancing of IPP. ${ }^{7}$ These four genes, together with $\operatorname{crt} Y$, were introduced into pRSET-A and $\mathrm{pET} 22 \mathrm{~b}(+)$ resulted in multicistronic operon vectors pR-IEIBY and pET22-iEIBY, respectively. Secondly, differ from other previous publications, another source of genes encode for the bottom mevalonate pathway enzymes including mvaK1, mvaK2, and $m v a D$ were cloned from Enterococcus faecium VTCC- B-935 isolated in Vietnam and introduced into pET28 vector forming pET28-mvaK1K2D. Subsequently, this vector was transformed into a recombinant $E$. coli strain which has already contained another expression vector pR-IEIBY. Finally, four additional carbon sources were investigated for higher production of $\beta$-carotene using our recombinant system.

\section{Materials and methods}

\section{Bacterial strains, primers, plasmids, and culture con- ditions}

Bacterial strains, primers, and plasmids used in this study are listed in Table 1. E. coli DH5 $\alpha$ was used for molecular cloning of target genes. E. coli BL21(DE3) was used for expression of mevalonate and carotenoid genes and subsequently the expressed recombinant enzymes produced $\beta$-carotene. Enterococcus faecium VTCC-B-935 and Pantoea ananatis DSM-17873 were used as donors of target genes. Primer sequences were designed based on nucleotide sequences of target genes available in NCBI database, D90087.2 for crtY and AF290095.1 for mva genes. Backbone plasmids including pLUG, pRSET-A, pET22b(+) and pET28a $(+)$ were used for gene cloning and recombinant enzyme expression.

Cultures of $E$. faecium and $P$. ananatis were performed following provider's instructions. For $\beta$-carotene production, recombinant $E$. coli clones were grown on LB agar plates under selection pressure of ampicillin $(100 \mu \mathrm{g} / \mathrm{mL})$ or combination of ampicillin/kanamycin $(50 \mu \mathrm{g} / \mathrm{mL})$ in an incubator at $37^{\circ} \mathrm{C}$ overnight. Individual colonies on plates were inoculated in a flask containing $5 \mathrm{ml}$ of LB media supplemented with the same antibiotic for $12 \mathrm{~h}$ in a rotary-shaking incubator at 180RPM. Subsequently the primary culture was transferred into $250 \mathrm{~mL}$ flask containing $50 \mathrm{~mL}$ LB media under the same condition. IPTG induction, at different concentrations, was performed while OD600nm reached 0.3-0.5 and continue inoculation for desired periods. Conditions were modified in optimization experiments according to planed changes. For biosynthesis of IPP and DMAPP by bottom portion of MVA pathway, mevalonate was add to the culture at $6.6 \mathrm{mM}$ as substrate. ${ }^{13}$

\section{Cloning of crtY, mvaKI, mvaK2, and mvaD genes}

Genomic DNA of $P$. ananatis and E. faecium were extracted using common protocols. Target genes were amplified using standard protocols and specific primers listed in Table 1. PCR products were subsequently ligated into $\mathrm{pLUG}$ cloning vector (Intron Biotechnology) by T4 DNA ligase (New England Biolabs). Ligation products were transformed into competent $E$. coli DH5 $\alpha$ (Thermo Fisher Scientific) cells by heat shock and plated onto LB agar plates supplemented with ampicillin $(100 \mu \mathrm{g} / \mathrm{mL})$, X-gal $(20 \mu \mathrm{g} / \mathrm{mL})$ and IPTG $(1 \mathrm{mM})$. White colonies were picked and inoculated in $5 \mathrm{ml}$ LB media. After extraction, plasmids were screened by size separation by agarose electrophoresis, and then mapped by appropriate restriction enzymes. Target inserts in positive clones were sequenced by Marcogen. Gene sequences were in silico analyzed by Vector NTI software to ensure correct translation, and aligned to available database using BLAST.

\section{Construction of recombinant plasmids}

In our previous study, four genes including idi, crtE, crtI, and crtB were introduced into pRSET-A resulted in pR-IEIB which was used for biosynthesis of Lycopene. ${ }^{7}$ In this study, cloned crtY gene was excited from pLUG-crtY and inserted into pR-IEIB using HindIII restriction enzyme resulted in pR-IEIBY. Subsequently, the multicistronic operon in pR-IEIBY containing five genes was moved to $\mathrm{pET} 22 \mathrm{~b}(+)$ by two steps. First, only crtY was moved using EcoRI and HindIII resulted in pET22-Y. Second, the fragment containing four genes idi, crtE, crtI and crtB was moved to pET22-Y using XbaI and EcoRI resulted in pET22-iEIBY. Similar experimental procedure was performed to introduce mvaK1, mvaK2, and mvaD into pET28a (+) to construct pET28-K1, pET28-K1K2, and pET28K1K1D at the positions of BamHI/EcoRI, EcoRI/SacI, and SacI/NotI, respectively. All vectors constructed in this study are listed in Table 1 and the maps of three major vectors are presented in Figure 2.

Where, pR-EIBY and pET22-IEIBY are pRSET-A and pET22b(+) 
containing multicistronic operon of five genes including idi from E. coli DH5 $\alpha$, crtE, crtI, crtB, and crtY from Pantoea ananatis, respectively. These two vector were used for biosynthesis of $\beta$-carotene in recombinant $E$. coli BL21(DE3). pET28-K1K2D is $\mathrm{pET} 28 \mathrm{a}(+)$ containing multicistronic operon of three genes including
mvaK1, mvaK2, and mvaD from Enterococcus faecium VTCC-B-935. This vector was used for conversion of mevalonate to IPP. Major component of the vectors and restriction sites used in the construction were indicated. Vector maps were drawn using Vector NTI software version 11.5.1.

Table I Bacterial strains, primers, and plasmids used in this study

\begin{tabular}{|c|c|c|}
\hline Name & Description & Reference/source \\
\hline \multicolumn{3}{|l|}{ Bacteria strains } \\
\hline E. coli DH5 $\alpha$ & TA cloning host & Thermo Fisher Scientific \\
\hline E. coli BL2 I (DE3) & Expression host compatible with vectors driven by $\mathrm{T7}$ system & Thermo Fisher Scientific \\
\hline $\begin{array}{l}\text { Pantoea ananatis } \\
\text { DSM-I7873 }\end{array}$ & Carotenoid genes donor & $\begin{array}{l}\text { German Collection of } \\
\text { Microorganisms and Cell } \\
\text { Cultures }\end{array}$ \\
\hline $\begin{array}{l}\text { Enterococcus } \\
\text { faecium } \\
\text { VTCC-B-935 }\end{array}$ & Mevalonate genes donor, isolated in Vietnam & $\begin{array}{l}\text { Vietnam Type Culture } \\
\text { Collection }\end{array}$ \\
\hline \multicolumn{3}{|l|}{ Primers* } \\
\hline mvaKI-F & ggatccATGGCAAACTATGGCCAAG & This study \\
\hline mvaKI-R & gaattcTTAAACATAGGTATGTACTCCT & This study \\
\hline mvaK2-F & gaattcATGATTGAAGTATCTGCACCA & This study \\
\hline mvaK2-R & gagctcTCATCGGTTTTCCTTTCTTTGA & This study \\
\hline mvaD-F & gagctcATGTTTAAAGGCAAAGCACG & This study \\
\hline mvaD-R & gcggccgcTTATTCAATAATCGCAATTCCTG & This study \\
\hline crtY-F & TgaattcAGGAGGTGTCTTAAATGGGAGCGGCTAT & This study \\
\hline crtY-R & GCaagcttTTAACGATGAGTCGTCATAATGG & This study \\
\hline \multicolumn{3}{|l|}{ Plasmids } \\
\hline PLUG & TA cloning vector & Intron Biotechnology \\
\hline pET28a $(+)$ & $\begin{array}{l}\text { Expression vector for low copy number (pBR322 ori), strong phage promoter } \\
\text { (T7), IPTG induction (lac operon), and Kanamicin selection (Kan') }\end{array}$ & Novagen \\
\hline pET22b(+) & $\begin{array}{l}\text { Expression vector for low copy number (pBR322 ori), periplasmic localization } \\
\text { (pelB signal peptide), strong phage promoter (T7), IPTG induction (lac operon), } \\
\text { and Ampicilin selection (Amp') }\end{array}$ & Novagen \\
\hline PRSET-A & $\begin{array}{l}\text { Expression vector for high copy number (pUC ori), strong phage promoter (T7), } \\
\text { IPTG induction (lac operon), and Ampicilin selection (Ampr) }\end{array}$ & Thermo Fisher Scientific \\
\hline pR-iEIB & pRSET-A containing idi of E. coli and crtE, crtl and $\mathrm{crtB}$ of $P$. ananatis & [7] \\
\hline pR-iEIBY & PRSET-A containing idi of E. coli and crtE, crtl, crtB, and $\mathrm{crtY}$ of $P$. ananatis & This study \\
\hline PET22-Y & $\mathrm{pET} 22 \mathrm{~b}(+)$ containing $\mathrm{crt} Y$ of $P$. ananatis & This study \\
\hline pET22-iEIBY & pET22 containing idi of E. coli and crtE, crtl, crtB, and $\mathrm{crtY}$ of $P$. ananatis & This study \\
\hline PET28-KI & $\mathrm{pET} 28 \mathrm{a}(+)$ containing mvaKI of E. faecium & This study \\
\hline PET28-KIK2 & $\mathrm{pET} 28 \mathrm{a}(+)$ containing $m v a K I$ and $m v a K 2$ of $E$. faecium & This study \\
\hline PET28-KIK2D & $\mathrm{pET} 28 \mathrm{a}(+)$ containing $m v a K I$, mvaK2, and mvaD of $E$. faecium & This study \\
\hline
\end{tabular}

(*) Template binding sequences are indicated by underlined letters, start and stop codons are bold, and restriction sites are lowercase. In cases of no restriction sites over hanged, autonomous sites in the carrying vectors were used instead.

Citation: Nam VH, Trang HHT, Huong TLT, et al. Enhanced $\beta$-carotene biosynthesis in recombinant Escherichia coli harboring the bottom portion of mevalonate pathway of Enterococcus faecium VTCC-B-935 isolated in Vietnam.J Appl Biotechnol Bioeng. 2017;3(4):374-379. DOI: 10.15406/jabb.2017.03.00073 

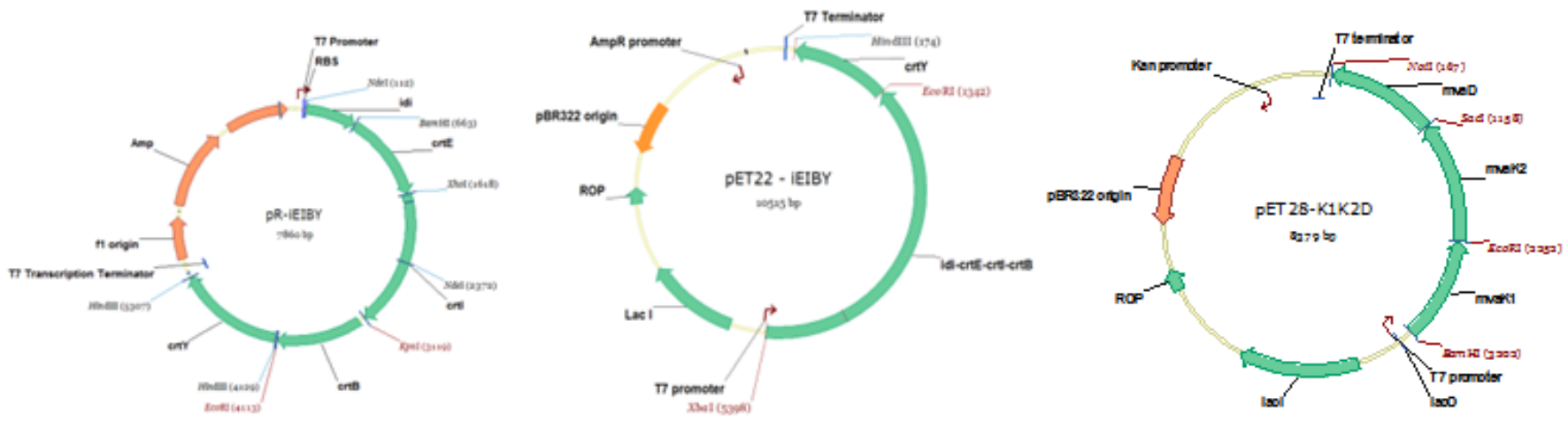

Figure 2 Expression vectors used for biosynthesis of $\beta$-carotene in this study.

\section{Measurement of $\beta$-carotene production}

Concentration of $\beta$-carotene synthesized by recombinant $E$. coli was measured by HPLC according to the protocol previously described by Yoon et al. ${ }^{12}$ with slight modifications. E. coli cells were harvested by centrifugation at $12,000 \mathrm{rpm}$ and washed once by $\mathrm{ddH}_{2} \mathrm{O}$. Cell pellet was re-suspended in $1 \mathrm{~mL}$ of acetone and incubate at $55^{\circ} \mathrm{C}$ for 10 to 15 minutes in dark condition. The extracted acetone containing $\beta$-carotene was collected by centrifugation at $12,000 \mathrm{rpm}$ for 15 minutes. HPLC analysis was performed on the Agilent 1260 system using Zorbax C18 column $(250 \mathrm{~mm} \times 4.6 \mathrm{~mm}, 5$ micromet $)$ with detector at $454 \mathrm{~nm}$. Two mobile phases were used including methanol (phase $A$ ) and acetonitrile (phase $B)$. Isocratic program $(A: B=70: 30)$ and flow rate of $1 \mathrm{ml} / \mathrm{min}$ was used to all analyses. Standard $\beta$-carotene was purchased from Sigma (Cat.No. C4582).

\section{Results and discussion}

\section{Comparison of pRSET-A and pET22b(+) for biosynthe- sis of $\beta$-carotene in engineered $E$. coli}

Different expression vectors produce different levels of desired recombinant proteins; in this case the five enzymes involved in the biosynthesis of $\beta$-carotene, and subsequently affect production of this carotenoid pigment. Therefore, we first compare two of the most common commercially available expression systems including pRSET-A and pET22b $(+)$. Although these two vector systems are both driven by $\mathrm{T} 7$ promoter, they are significantly different in term of copy number. In case of carotenoid production by recombinant $E$. coli, contradictory observations regarding the biosynthesis efficiency caused by plasmid copy number were reported. Jones et al. ${ }^{22}$ showed equal or better performance of low copy plasmids in comparison to high copy plasmids. ${ }^{22}$ However, using a mutated version of the low copy vector $\mathrm{pDC} 318$ that made it a high copy one, Tao et al. ${ }^{23}$ observed that mutated vector showed higher level of $\beta$-carotene biosynthesis. ${ }^{23}$ In our study, accumulation of $\beta$-carotene was higher with pRSET-A than pET22b $(+)$ according to higher density of yellow color of the cell pellet (Figure 3). HPLC analysis confirmed the biosynthesis of $\beta$-carotene by $\mathrm{pR}$-IEIBY and $\mathrm{pET} 22$-iEIBY are $5.5 \mathrm{mg} / \mathrm{L}$ and $3.1 \mathrm{mg} / \mathrm{L}$, respectively. pRSET-A harbor pUC origin of replication which manages approximately 500 copies of the vector per single cell. This number is about 10 to 20 folds higher than what driven by pBR322 origin of replication located in pET22b(+). Although pET22b(+) and pRSET-A are not totally identical but they are both driven by T7 promoter. Therefore, in term of plasmid copy number our data is in agreement with Tao et al. ${ }^{23}$

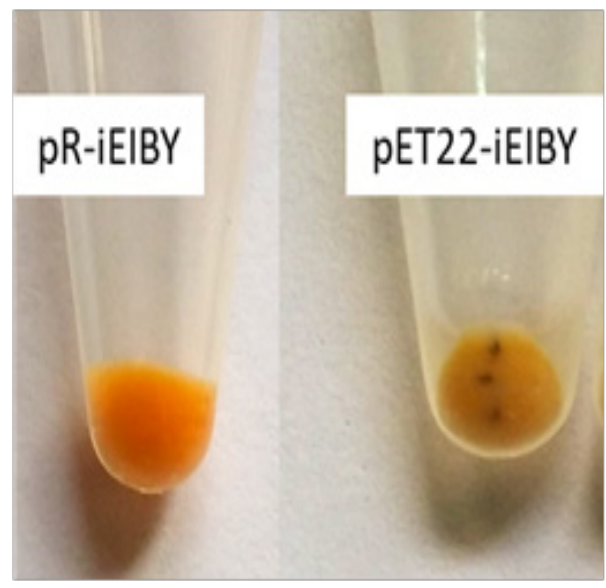

Figure 3 Comparison of pRSET-A and pET22b(+) for biosynthesis of $\beta$-carotene in recombinant $E$. coli $\mathrm{BL2}$ I (DE3).

Higher accumulation of $\beta$-carotene in cell pellet of pR-IEIBY than in PET22IEIBY was demonstrated by higher density of yellow color which is the color of this pigment.

\section{Increased production of $\beta$-carotene by addition of bo- ttom portion of mevalonate pathway}

Isopentenyl diphosphate (IPP) and dimethylallyl diphosphate (DMAPP) are precursors of all carotenoid, including $\beta$-carotene. Therefore, to enhance production of $\beta$-carotene, it is reasonable to direct the biosynthesis flow of the $E$. coli host toward these building blocks via either endogenous MEP pathway or exogenous MEV pathway, or both (Figure 1). Several studies have proven the feasibility of this strategy. ${ }^{6,12,13,18}$ In genetic engineering studies focusing on production of recombinant proteins, it is common that different sources of target genes resulted in different level of expression because of the suitability between genetic code of foreign genes with the genetic reading system/mechanism of specific host. In this study, we test another set of bottom portion of MVA pathway derived from E. faecium isolated in Vietnam.

E. coli BL21 (DE3) harboring pR-IEIBY was made competent. This enabled second transformation with pET28-K1K2D resulted in a recombinant strain harboring both carotenoid vector and MVA vector, in this case are pR-IEIBY and pET28-K1K2D, respectively. E. coli culture was supplied with mevalonate at $6.6 \mathrm{mM}$ which the optimal concentration is shown by Yoon et al. ${ }^{13}$ It is clear that addition of bottom portion of MVA pathway of E. faecium VTCC-B-935 did not 
affect growth of E. coli (Figure 4A). This observation is in agreement with several previous reports. ${ }^{6,11-13}$ To date, $\beta$-carotene has not been shown as a toxic agent to $E$. coli. As expected, over expression of three mva genes significantly up regulates the biosynthesis of $\beta$-carotene (Figure 4B). At 48 hours and 96 hours of cultivation, addition of bottom MVA pathway derived from E. faecium VTCC-B-935 resulted in $12.6 \mathrm{mg} / \mathrm{L}$ and $17.7 \mathrm{mg} / \mathrm{L}$ of $\beta$-carotene which are approximately three folds higher than $4.2 \mathrm{mg} / \mathrm{L}$ and $5.6 \mathrm{mg} / \mathrm{L}$, respectively, produced in control strain. This result indicated that the three genes, mvaK1, mvaK2, and mvaD of E. faecium VTCC-B-935 are comparable to the corresponding genes of Streptococcus pneumoniae in term of IPP biosynthesis in recombinant $E$. coli. ${ }^{13}$

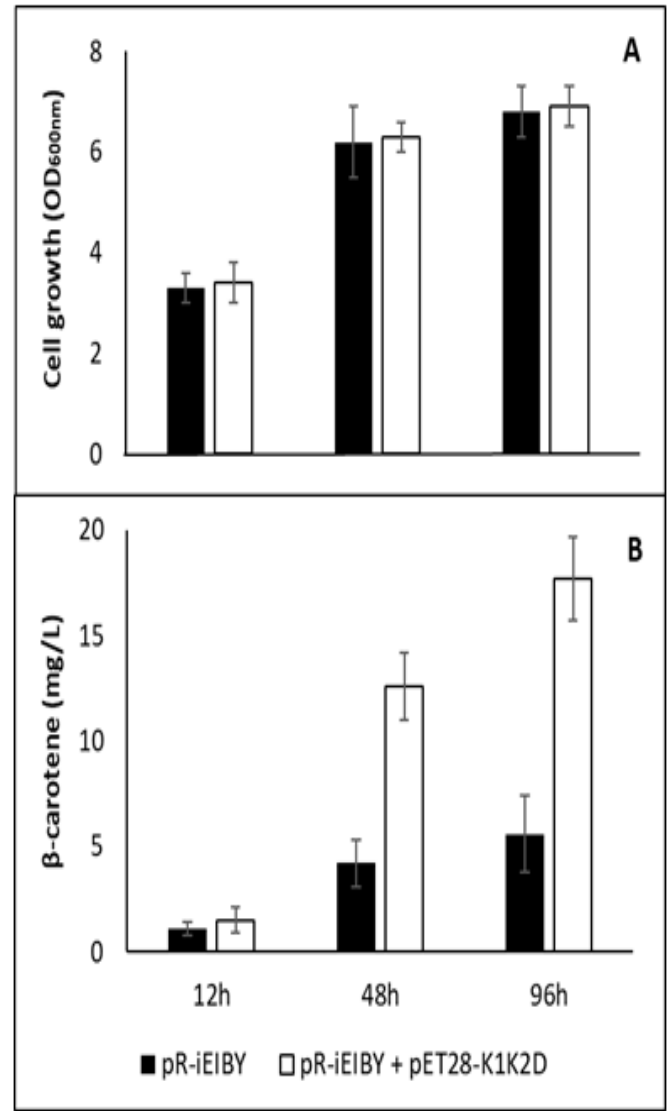

Figure 4 Cell growth and $\beta$-carotene production of recombinant $E$. coli with or without addition of bottom portion of MVA pathway from $E$. faecium VTCC-B-935.

PR-IEIBY contains $\beta$-carotene biosynthesis genes and idi. PET28-KIK2D contains genes encoding enzymes catalyze reactions from mevalonate to IPP.

\section{Effect of addition of carbon sources on $\beta$-carotene pro- duction}

Carbon source was determined as one of the factors related to biosynthesis of $\beta$-carotene. Insufficient availability of carbon sources restricts the growth of cell. Again, contradictory observations were reported regarding the effect of different carbon sources on biosynthesis of carotenoid by recombinant E. coli, including $\beta$-carotene. Biosynthesis of toulene was shown increased and decreased following addition of glycerol and glucose, respectively. ${ }^{24}$ Contradictorily, production of lycopene was significantly enhanced by addition of glucose in high cell density fed-batch fermentation conditions. ${ }^{25}$
In this study, four sources of carbon were investigated, including glucose, galactose, maltose, and glycerol. The optimal concentration is $0.5 \%$ according to Yoon et al. ${ }^{12}$ Therefore, we added $0.5 \%$ of each of carbon sources to the $2 \mathrm{YT}$ medium. In Figure 5, the positive correlation between cell growth and $\beta$-carotene yield was observed. Among the tested carbon sources, glycerol indicated significantly improvement in both cell growth and $\beta$-carotene production over the others. In this case, the highest yield of $\beta$-carotene was $65 \mathrm{mg} / \mathrm{L}$ which was approximately three fold higher than the control which did not contain additional carbon source. Beside glycerol, addition of maltose slightly improved cell growth and $\beta$-carotene production. However, addition of glucose and galactose resulted in lower cell growth and $\beta$-carotene biosynthesis compared to the control. One of the reasons to explain this phenomenon is that glucose and galactose decreased $\mathrm{pH}$ of the culture that leads to inhibition of $E$. coli growth.

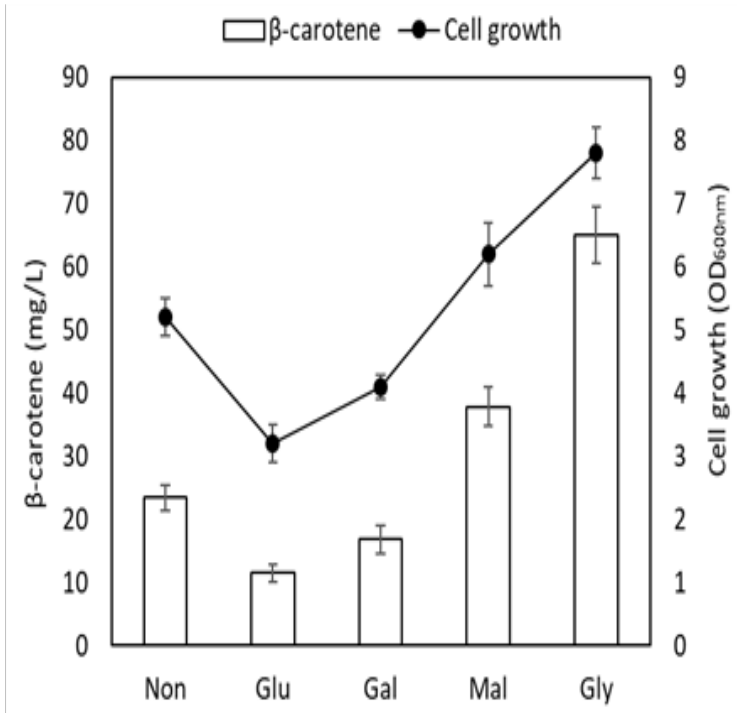

Figure 5 Effect of various carbon sources on growth and production of $\beta$-carotene by recombinant E. coli harboring PR-IEIBY and PET28-KIK2D.

Non, Control which did not contain any additional carbon source; Glu, glucose; Gla, galactose; Mal, maltose; Gly, glycero

\section{Conclusion}

The bottom portion of mevalonate pathway of E. faecium VTCC-B-935 isolated in Vietnam was introduced into a engineered carotenogenic $E$. coli resulted in improvement of $\beta$-carotene biosynthesis. The pRSET-A expression vector performed better than $\mathrm{pET} 22 \mathrm{~b}(+)$. Addition of glycerol as carbon source enhanced E. coli cell growth and biosynthesis of $\beta$-carotene almost 3 folds.

\section{Acknowledgements}

This work was supported by the Research Grant from the Vietnam Ministry of Education and Training (Grant No B2014-TN02-01).

\section{Conflict of interest}

The author declares no conflict of interest.

\section{References}

1. Milani A, Basirnejad M, Shahbazi S, et al. Carotenoids: biochemistry, pharmacology and treatment. Br J Pharmacol. 2017;174(11):1290-1324. 
2. Marz U. The Global Market for Carotenoids. USA: BCC Research LLC; 2008.

3. Mantzouridou F, Roukas T, Kotzekidou P. Production of beta-carotene from synthetic medium by blakeslea trispora in fed-batch culture. Food Biotechnology. 2004;18(3):343-361.

4. Bhosale P, Gadre RV. Beta-Carotene production in sugarcane molasses by a Rhodotorula glutinis mutant. J Ind Microbiol Biotechnol. 2001;26(6):327-332.

5. Hejazi MA, De Lamarliere C, Rocha JM, et al. Selective extraction of carotenoids from the microalga Dunaliella salina with retention of viability. Biotechnol Bioeng. 2002;79(1):29-36.

6. Yang J, Guo L. Biosynthesis of $\beta$-carotene in engineered E. coli using the MEP and MVA pathways. Microbial Cell Factories. 2014;13(1):160.

7. Sun T, Miao L, Li Q, et al. Production of lycopene by metabolically engineered E. coli. Biotechnol Lett. 2014;36(7):1515-1522.

8. Lemuth K, Steuer K, Albermann C. Engineering of a plasmid-free Escherichia coli strain for improved in vivo biosynthesis of astaxanthin. Microb Cell Fact. 2011;10:29.

9. Kim YS, Lee JH, Kim NH, et al. Increase of lycopene production by supplementing auxiliary carbon sources in metabolically engineered Escherichia coli. Appl Microbiol Biotechnol. 2011;90(2):489-497.

10. Jang HJ, Yoon SH, Ryu HK, et al. Retinoid production using metabolically engineered Escherichia coli with a two-phase culture system. Microb Cell Fact. 2011;10:59.

11. Chae HS, Kim KH, Kim SC, et al. Strain-dependent carotenoid productions in metabolically engineered Escherichia coli. Appl Biochem Biotechnol. 2010;162(8):2333-2344.

12. Yoon SH, Lee SH, Das A, et al. Combinatorial expression of bacteria whole mevalonate pathway for the production of beta-carotene in $E$. coli. J Biotechnol. 2009;140(3-4):218-226.

13. Yoon SH, Park HM, Kim JE, et al. Increased beta-carotene production in recombinant Escherichia coli harboring an engineered isoprenoid precursor pathway with mevalonate addition. Biotechnol Prog. 2007;23(3):599-605.

14. Yoon SH, Kim JE, Lee SH, et al. Engineering the lycopene synthetic pathway in E. coli by comparison of the carotenoid genes of Pantoea agglomerans and Pantoea ananatis. Appl Microbiol Biotechnol. 2007;74(1):131-139.
15. Pitera DJ, Paddon CJ, Newman JD, et al. Balancing a heterologous mevalonate pathway for improved isoprenoid production in Escherichia coli. Metab Eng. 2007;9(2):193-207.

16. Nishizaki T, Tsuge K, Itaya M, et al. Metabolic engineering of carotenoid biosynthesis in Escherichia coli by ordered gene assembly in Bacillus subtilis. Appl Environ Microbiol. 2007;73(4):1355-1361.

17. Yuan LZ, Rouvière PE, Larossa RA, et al. Chromosomal promoter replacement of the isoprenoid pathway for enhancing carotenoid production in E. coli. Metab Eng. 2006;8(1):79-90.

18. Yoon SH, Lee YM, Kim JE, et al. Enhanced lycopene production in Escherichia coli engineered to synthesize isopentenyl diphosphate and dimethylallyl diphosphate from mevalonate. Biotechnol Bioeng. 2006;94(6):1025-1032.

19. Yan Y, Chemler J, Huang L, et al. Metabolic engineering of anthocyanin biosynthesis in Escherichia coli. Appl Environ Microbiol. 2005;71(7):3617-3623

20. Vadali RV, Fu Y, Bennett GN, et al. Enhanced lycopene productivity by manipulation of carbon flow to isopentenyl diphosphate in Escherichia coli. Biotechnol Prog. 2005;21(5):1558-1561.

21. Kim SW, Keasling J. Metabolic engineering of the nonmevalonate isopentenyl diphosphate synthesis pathway in Escherichia coli enhances lycopene production. Biotechnol Bioeng. 2001;72(4):408-415.

22. Jones KL, Kim SW, Keasling JD. Low-copy plasmids can perform as well as or better than high-copy plasmids for metabolic engineering of bacteria. Metab Eng. 2000;2(4):328-338.

23. Tao L, Jackson RE, Cheng Q. Directed evolution of copy number of a broad host range plasmid for metabolic engineering. Metab Eng. 2005;7(1):10-17.

24. Lee PC, Mijts BN, Dannert CS. Investigation of factors influencing production of the monocyclic carotenoid torulene in metabolically engineered Escherichia coli. Appl Microbiol Biotechnol. 2004;65(5):538546.

25. Alper H, Miyaoku K, Stephanopoulos G. Characterization of lycopene-overproducing $E$. coli strains in high cell density fermentations. Appl Microbiol Biotechnol. 2006;72(5):968-974. 